\title{
LXXVI. On the constitution of the urine in man and carnivorous animals
}

\author{
Justus Liebig M.D. Ph.D. F.R.S. M.R.I.A.
}

To cite this article: Justus Liebig M.D. Ph.D. F.R.S. M.R.I.A. (1844) LXXVI. On the constitution of the urine in man and carnivorous animals, Philosophical Magazine Series 3, 25:168, 453-480, DOI: $10.1080 / 14786444408646084$

To link to this article: http://dx.doi.org/10.1080/14786444408646084

册 Published online: 30 Apr 2009.

Submit your article to this journal $\lceil\pi$

ЏII Article views: 2

Q View related articles $\square$ 
obscura; and where, from situation or otherwise, there is a difficulty in observing the colour of the plate during the process of iodizing, it may be removed from the iodine vessel and its colour examined by the direct light of the sun without risk of injury; for when returned to the iodine or bromine vessel for a moment the effect of light is wholly destroyed.

Perhaps the most valuable practical application of these facts is in the use of the same plate for receiving several impressions. When, on taking a portrait or the picture of any object liable to move, there is reason to suppose that the motion of the person or object has rendered the operation useless, it is not necessary to throw aside the plate on which the imperfect impression has been taken, and resort to the tedious process of cleaning and preparing another; it is only necessary to treat the plate in the manner already pointed out, and it is again equal in every respect to a newly prepared plate; and this treatment may be repeated until by the slow accumulation of too thick a film of iodide of silver, the plate no longer possesses the same degree of sensitiveness to light. Temple Row West, Birmingham, George Shaw. November $\mathrm{J} 5,1844$.

LXXVI. On the Constitution of the Urine in Man and Carnivorous Animals. By Justus Liebig, M.D., Ph.D., F.R.S., M.R.I.A., Professor of Chemistry in the University of Giessen*.

I $\mathrm{T}$ is a very curious and remarkable fact that all the analyses and examinations hitherto made of the urine of man and of carnivorous animals have not yet afforded us any satisfactory answer to the question, What is the substance which imparts to that fluid its property of reddening blue vegetable colours?

In most physiological and chemical works we find the acid reaction of urine ascribed either to uric acid or to lactic acid; but no positive and definite proof is given of the presence of the latter acid in urine.

The acidification of milk, that is, the formation of lactic acid, is dependent upon the milk-sugar contained in milk; this substance being in contact with caseine in a state of decomposition and transformation, undergoes, by its means, an alteration, which consists in its elements transposing and arranging themselves into lactic acid without the addition or separation of any one atom. Crystallized milk-sugar $\left(\mathrm{C}_{12} \mathrm{H}_{12} \mathrm{O}_{12}\right)$ and hydrate of lactic acid $\left(\mathrm{C}_{6} \mathrm{H}_{6} \mathrm{O}_{6}\right)$ have one and the same

* From the Lancet, Nos, xi. and xii., by the obliging permission of $T$. Wakley, Esq., M.P. 
composition. It is a well-known fact, that when all the milksugar in a given quantity of milk has become converted into lactic acid, a fresh quantity of lactic acid may be produced by repeated addition of milk-sugar; indeed, the common white caseine in a state of decomposition yields, upon renewed additions of milk-sugar, almost unlimited quantities of lactic acid, provided the precaution be taken to neutralise the free acid from time to time by means of an alkali. Grape-sugar and cane-sugar, under the same conditions, yield the same products as milk-sugar. The examinations and experiments of Haidlen* have proved that milk in its fresh state contains neither lactic acid nor lactates.

So long as chemists were ignorant of the intimate relation existing between lactic acid and the milk-sugar contained in milk, there appeared to be some reasons for believing in the presence of lactic acid in animal secretions generally, when manifesting an acid reaction, or passing into a state of acidity. It thus happened, without any real and positive proof, that lactic acid was considered to be a constituent of urine; the presence of lactates in the blood was assumed, and these salts were even supposed to perform a highly important part in the process of respiration.

Lactic acid is a non-nitrogenous substance. Nothing has hitherto been observed tending to show that it may be produced from the elements of a nitrogenous substance, by the decomposition of such a substance and the transposition of its elements. In every instance where the formation of lactic acid has been observed, the result of careful examination has proved the presence of a non-nitrogenous substance of an identical or, at least, similar composition with lactic acid.

These observations would seem to render the formation of lactic acid in the bocly of the herbivorous and graminivorous animals, which take starch and sugar in their food (substances from which lactic acid may be formed), not merely possible, but in many cases highly probable; and yet, strange to say, chemists have hitherto attempted in vain to detect lactic acid in the urine of the cow and of the horse. The urine of the cow or horse has no acid reaction; on the contrary, its reaction is strongly alkaline; it contains carbonated, hippurated, or benzöated alkali, or alkalies combined with mineral acids, but no trace of any lactate.

In contrast with this, the urine of man, and of carnivorous animals, manifests, when in a healthy state, a strongly acid reaction. Now, it is precisely in analyses of the blood and urine of man, and of carnivorous animals, that we find Jac-

* Annalen der Chemie und Pharmacie, vol. xi. p. 263. 
tates mentioned as constant constituents; not because they have in reality been detected in these fluids,-for no one has as yet succeeded in producing lactic acid therefrom,--but because, upon examining the aqueous and alcoholic extracts of blood and urine, some non-crystalline matters have been found which sometimes manifested an acid reaction, and upon incineration left a carbonated alkali as a residue, thus presenting a remote similarity in deportment to the alkaline lactates.

From what substance could lactic acid be formed in the body of carnivorous animals? With the exception of fat, they partake of no non-nitrogenous matter in food, no such substance, in fact, which is, so far as we know, capable of producing lactic acid. Carnivorous animals partake of no sugar, no starch, no gum, no mucus; there is a total absence of the non-nitrogenous substances which form so large a part of the aliments of herbivorous and graminivorous animals.

The assumption, à priori, that neither the blood nor any other fluid in the body of camivorous animals can possibly contain any lactic acid, has been positively established by the experiments of Enderlin*. Finally, Pelouze has proved that the experiments of Heury, who pretended he had detected lactate of urea in urine, are erroneous, and by no means to be relied upon.

Consequently, as our knowledge of this subject stands at present, the acid reaction of urine cannot proceed from lactic acid. And although processes of transposition take place in the healthy animal body, rendering insoluble substances soluble in the stomach and bowels, yet these are of a different kind from that process of putrefaction of caseine in milk which produces lactic acid from the elements of the non-nitrogenous elements.

Direct experiments prove that fresh urine, of a strongly acid reaction, and taken from various healthy individuals, when cautiously neutralized with water of barytes, does not retain in solution the least detectable trace of barytes. Now, as lactate of barytes is readily soluble in water, the urine would certainly, and of necessity, contain barytes, if its acid reaction were really owing to the presence of lactic acid. Upon the addition of the very first drop of water of barytes to urine a copious precipitate is formed; this precipitate contains urate and phosphate of barytes and of lime, and no detectable trace of barytes is found, even although only just so much water of barytes is added as to leave the urine still possessing a feebly acid reaction in the solution filtered from the precipitate.

Carbonate of magnesia and calcined magnesia act upon

* Annalen der Chemie und Pharmacie, vol. xlix. p. 317, and vol. l. 
urine in precisely the same manner. If either of these substances be mixed with water, so as to form a milky fluid, and be then added to urine with an acid reaction, the acid reaction immediately disappears, and a very considerable white precipitate is formed. The fluid now manifests a feebly alkaline reaction, and contains a trace of magnesia in solution. It is a remarkable circumstance that magnesia withdraws the phosphoric acid from the urine so completely, that a mixture of perchloride of iron and acetate of potass no longer indicates a trace of phosphoric acid in the urine which has thus been treated with magnesia.

Had lactic acid been the solvent of the phosphate of lime and magnesia in the urine, one would have expected that a corresponding amount of barytes, or of magnesia, would have taken its place upon its separation. But, as I have already observed, not a trace of barytes is found in solution when barytes has been employed for neutralizing the acid, and only a slight trace of magnesia when the latter substance has been used for the same purpose.

But, as urine contains a certain amount of alkaline phosphates, i. e. phosphate of soda and phosphate of potass, and as barytes and magnesia form, with phosphoric acid, insoluble salts, it might have been supposed that the neutral lactates formed upon the neutralization of the urine with the two bases had been decomposed, together with the phosphates of soda and potass contained in the urine, and transposed themselves anew, with these substances, into phosphate of barytes or of magnesia, and into neutral lactate of potass or soda. In this case neither barytes nor magnesia could remain in solution. This circumstance, therefore, renders these experiments indecisive, and leaves the question as to the presence or absence of lactic acid in urine dependent upon more direct experiments.

I employed putrid urine in my attempts to detect lactic acid, because lactic acid is not destroyed by putrefaction, and it must, therefore, of necessity, be present in putrid urine if it really forms a constituent of fresh urine; and because, if lactic acid can at all be formed by the putrefaction of urine, from matters containing previously no lactic acid, the question whether lactic acid is to be reckoned among the constituents of normal urine is at once practically decided ; or, more correctly speaking, the problem is proved to be impossible of solution, since we possess no means of positively determining which urine may be considered of a normal constitution, and, on the contrary, which is to this extent abnormal. As matters at present stand, therefore, with regard to this subject, it 
was immaterial whether the presence of Jactic acid was detected in fresh or in putrid urine; if it was found to exist in the latter, this fact must be considered as a confirmation of Berzelius's examination of fresh urine; whilst its absence from putrid urine would justify us positively to assert that it does not form a constituent of fresh urine; and, moreover, that urine contains no substance giving origin, by putrefaction, to the formation of lactic acid.

1 have come to the latter conclusion. I found it impossible to detect the presence of lactic acid in putrid urine; and if we examine somewhat more closely and minutely the experiments made by Berzelius, and from which he inferred the presence of lactic acid in urine, we find that not one of them amounts to a positive proof that lactic acid really forms a constituent of fresh urine.

The experiments which I made for the purpose of ascertaining the presence of lactic acid in putrid urine are the following :-

Putrid urine was first evaporated over an open fire, and afterwards to dryness in a water-bath; the residue was treated with a mixture of alcohol and sulphuric acid, which caused the solution of phosphoric acid, hydrochloric acid, and of lactic acid also, if this latter substance were really present. The fluid obtained was saturated with oxide of lead, and then filtered off from the phosphate, sulphate, and chloride of lead formed; the lead contained in the filtered solution was separated by sulphuretted hydrogen. The solution thus freed from lead, and which ought to have contained the lactic acid, had there been any present, was evaporated in a water-bath, and the residue treated with alcohol, - a quantity of common salt remained. In order to remove the soda from the alcoholic solution, effloresced oxalic acid was dissolved in the latter, at a high temperature, and the oxalate of soda formed was separated from the fluid by filtration; the fluid was then saturated with oxide of lead, which again gave rise to the formation and separation of chloride of lead. The solution was again freed from the lead which had dissolved sulphuretted hydrogen, then concentrated in the water-bath, and basic acetate of lead added in excess; a copious white precipitate was formed, from which the fluid was filtered off. This fluid must contain the lactic acid if any had been present in the urine; the lead which this fluid held in solution was precipitated by sulphuretted hydrogen, the fluid filtered off from the precipitate, concentrated in the water-bath and boiled with bydrate of barytes, - a quantity of ammonia was expelled by this 
operation. After the decomposition of the ammoniacal salt the new-formed salt of barytes was cautiously decomposed by sulphate of zinc, and every possible means was applied to obtain from this fluid crystals of lactate of zinc, but without success-no trace could be discovered.

The white precipitate obtained by means of the basic acetate of lead contained hydrochloric acid, and a brown resinous substance, which, upon combustion, behaved like an animal substance.

In other experiments the putrid urine was boiled until all the carbonate of ammonia it contained was completely expelled; then, with addition of hydrate of lime to destroy the remaining salts of ammonia, evaporated to dryness, and the residue treated with cold water, which must have dissolved lactate of lime had any lactic acid been present in the urine. The aqueous extract was evaporated to dryness, and the residue again treated with alcohol; the fluid obtained contained a copious amount of lime combined with an organic acid; the lime was then removed by the addition of oxalic acid, and the excess of oxalic acid by the addition of oxide of lead; the minute trace of oxide of lead which dissolved was removed by means of charred blood. The fluid obtained was very acid; it contained hydrochloric acid, which was removed by the addition of oxide of silver; a portion of the fluid filtered off from the hydrochlorate of silver formed was saturated with oxide of zinc, and left to crystallize, but no lactate of zinc was obtained; the fluid settled into a dark-coloured resinous mass. Another portion of this acid fluid was evaporated in the water-bath; a quantity of acetic acid was expelled during the evaporation, and there remained at last only a very minute amount of a resinous matter, which upon calcination emitted a very fetid odour.

All the other experiments which I made in order to cletect lactic acid in putrid urine, and a detailed description of which would be as tedious as useless, gave the same negative result. These experiments were usually made upon quantities of from forty to fifty pounds of urine, so that even a very minute amount of lactic acid, if really present in the urine, could not have escaped detection. All these experiments indicated the presence of an organic acid, but after the removal of all the inorganic acids and bases contained in the urine, this acid turned out to be a mixture of acetic acid with a brown resinous substance rich in nitrogen.

To remove any doubt on this point I took putrid urine, freed it from its carbonate of ammonia by evaporation, and 
subnitted it to distillation with dilute sulphuric acid, hydrochloric acid, and oxalic acid; I obtained so much acetic acid as to be able to prepare with it many ounces of acetate of lead; from the salt of lead thus formed I was able to produce acetic æther and concentrated acetic acid, which, by mere agitation with ather, lost all urinous odour. Over and above all these tests, the salt of silver formed by this acid was subjected to analysis; it had crystallized like the common acetate of silver, in shining scales, of easy solution in hot water.

$0.4319 \mathrm{grm}$. of this salt of silver yielded $0.2808 \mathrm{grm}$. of metallic silver.

$0.9375 \mathrm{grm}$. yielded 0.4737 of carbonic acid, and 0.1508 of water.

According to this analysis it consists of,-

\begin{tabular}{|c|c|}
\hline & $\begin{array}{r}\text { Found. } \\
13.89\end{array}$ \\
\hline & \\
\hline gen & $1 \cdot 78$ \\
\hline Oxy & $19 \cdot 32$ \\
\hline Silver & $65^{\circ} 01$ \\
\hline
\end{tabular}

and this agrees perfectly with the known composition of acetate of silver.

With respect to the presence of acetic acid in putrid urine, this was positively proved by Proust, in a paper published about twenty-four years ago*, and has subsequently been confirmed by Thenard. This paper of Proust, as well as all the facts and experiments mentioned in it, seems to have escaped altogether the attention of those chemists who have been subsequently engaged in investigating the composition of urine. What I have to add to and correct in these works is, in part, contained in Proust's paper.

Thus, for instance, Pronst observed that in the distillation of urine with sulphuric acid or hydrochloric acid, a certain amount of benzoic acid passes over together with acetic acid, and is deposited in crystals in the neck of the retort. I have found that, upon saturating with oxide of lead the acetic acid obtained in this process, a considerable amount of a white precipitate is formed, consisting of pure benzoate of lead. Moreover, if the concentrated putrid urine is mixed with some sulphuric acid, and allowed to stand at rest for the space of several days, a quantity of benzoic acid is obtained in shining brown scales; none of the various urines submitted to these experiments were free from this acid.

$0.3544 \mathrm{grm}$. of the benzoic acid produced from urine yielded upon combustion 0.8805 of carbonic acid and 0.1618 of water. This gives in 100 parts-

* Annales de Chimie et de Physique, vol. xiv. p. 260. 


\begin{tabular}{|c|c|c|}
\hline Carbon & $\begin{array}{l}\text { Found. } \\
\text {. } 68.58\end{array}$ & $\begin{array}{c}\text { Calculated. } \\
69 \cdot 10\end{array}$ \\
\hline Hydrogen & $\begin{array}{r}.07 \\
. \quad 5.07\end{array}$ & $4 \cdot 87$ \\
\hline Uxygen & - $26 \cdot 35$ & $26 \cdot 03$ \\
\hline
\end{tabular}

These proportions correspond exactly with the formula of benzoic acid.

With respect to the question of the presence of acetic acid and benzoic acid in fresh urine, no doubt can be entertained that the latter is not contained, as benzoic acid, in fresh urine, since it has been proved by the experiments of Ure and Keller, that crystallized benzoic acid becomes in the organism converted into hippuric acid, and appears in the urine as hippurate of soda. Now, as we also know that the hippuric acid contained in the urine of herbivorous animals passes into decomposition upon the putrefaction of their urine, and that benzoic acid is one of the products of this decomposition, we may safely and positively assume that the benzoic acid in putrid human urine has the same origin, and that consequently it must exist in fresh urine in the form of hippuric acid. This has been fully confirmed by the results of minute examinations. All the urine taken in this country from individuals living upon a mixed animal and vegetable diet, contains hippuric acid, besides uric acid, and about the same proportion of both acids. Hippuric acid may be obtained in the following manner, even from proportionally small amounts of fresh urine :-Fresh urine is evaporated in a water-bath to the consistence of a syrup; it is then mixed with some hydrochloric acid, and agitated with its own volume of æther, which latter substance dissolves the hippuric acid. It usually happens that the mixture does not separate spontaneously, but that the xther remains inclosed by the fluid, like froth; the separation of the æether takes place immediately upon adding to the mixture, after having allowed it to stand for an hour, one-twentieth part of its volume of alcohol. In this case the froth disappears, and the fluid separates into two layers; the upper layer contains the hippuric acid in solution, but it also contains urea, owing to the addition of the alcohol. This upper layer is carefully removed by means of a pipette or siphon, and agitated with small portions of water; the water removes the alcohol and the urea, whilst the hippuric acid remains in solution in the æther. By evaporating the æthereal solution the hippuric acid is obtained in crystals, which are usually of a yellowish or brown colour, imparted to them by the presence of a resinous substance, which may be easily and completely removed by means of charred biood.

In its pure state the hippuric acid obtained from human 
urine presents the same long, shining, transparent, four-sided, obliquely-truncated prisms, by which the hippuric acid procured from the urine of animals is so easily detected and distinguished from benzoic acid. The hippuric acid of human urine is not volatile at the subliming temperature of benzoic acid; at a higher temperature it undergoes fusion, forming a brown-red liquid, and yields upon dry distillation the same products which common hippuric acid forms under the same circumstances, viz. a red-coloured oil, smelling like tonkabeans, ammonia, benzoic acid, and a copious residue of carbon. It dissolves in nitric acid at a high temperature, and yields, upon cooling, crystals of benzoic acid, owing to the decomposition which it undergoes.

From $0.499 \mathrm{grm}$. of hippuric acid produced from urine, 1.0791 of carbonic acid and 0.2317 of water were obtained. This gives for 100 parts-.

$$
\begin{array}{llcc} 
& & \text { Found. } & \text { Calculated. } \\
\text { Carbon } & \text {. } & 59 \cdot 47 & 60 \times 89 \\
\text { Hydrogen } & - & 5 \cdot 15 & 4 \times 45
\end{array}
$$

This analysis corresponds sufficiently with the calculated results to remove all doubt as to the nature of this acid; it will be perceived that it contains 10 per cent. less carbon than benzoic acid.

As far as our investigations into the composition of the aliments of man will allow us to judge, they contain no benzoic acid from which hippuric acid might have been formed, and as the urine of cows is invariably rich in hippuric acid, no matter whether the cows have been fed upon hay or upon beet-roots,--of which latter plants we know positively, from the results of several examinations, and from experience derived from the manufacture of beet-root sugar, that they contain no benzoic acid,-we can come to no other conclusion concerning the presence of hippuric acid in the urine of the herbivorous animals, and in the urine of persons living upon a mixed vegetable and animal diet, than that it is a product formed in the organism, to the formation of which the elements of their non-nitrogenous aliments give birth.

The presence of acelic acid in putrid urine does not warrant us to infer that this acid is also present in fresh urine; on the contrary, the experiments made with regard to this matter prove that fiesh urine contains no acetic acid. I have treated fresh urine exactly in the same manner as putrid urine, and have by distillation with oxalic acid, for instance, obtained a fluid of a strongly resinous odour, but not possessing any 
acid reaction. When employing sulphuric acid and hydrochloric acid the distillate was acid, but the acid reaction proceeded from hydrochloric acid.

I have, in the preceding experiments, several times alluded to a nitrogenous resin-like substance, which is present in all the fluids obtained in the examination of putrid urine. This substance is a product of the putrefaction of the urine, and is of an acid nature. If the residue remaining upon the evaporation of putrid urine is treated with alcohol, and the resulting solution boiled with addition of hydrate of lime, until ammonia ceases to be given off, then evaporated to dryness, and the residue treated with cold alcohol, there remains a mass of a light brown colour, which, at a high temperature, may be kneaded like resin, and drawn out into long threads, and which, upon combustion, emits a strong smell of urine, and leaves a quantity of carbonate of lime as ashes.

If putrid urine is evaporated and sulphuric acid added, after the lapse of some time, an oily, black-coloured substance separates, which, being washed with water, assumes the appearance of pitch. It is soluble in alcohol and in alkalies. Upon distilling putrid urine with mineral acids this substance is obtained in the residue to a considerable amount, although altered in many of its properties. A portion of it, produced in this manner, has, - apparently from the action of the acids at a high tempernture, - completely lost its solubility in alcohol, whilst its solubility in alkalies remains unimpaired. From this alkaline solution it may again be precipitated in black flakes by acids. Proust has described these substances minutely, but as they cannot be procured from fresh urine, they are, as products of the putrefaction of urine, of no interest in the present investigation.

Just as benzoic acid and a nitrogenous substance are products of the putrefaction of hippuric acid in urine, I consider acetic acid and the above-mentioned nitrogenous resinous substance to stand in a definite relation to each other; that is, I consider them to be products of the decomposition of a substance containing the elements of both; and this substance seems to be no other than the colonring matter of the urine. Not the slightest trace of alcohol has ever been detected in urine excreted after the use of spirituous liquors. It is evident, therefore, that the acetic acid present in putrid urine cannot owe its origin to the use of spirituous liquors. If sugar, or sugar of milk, be added to fresh urine, and the urine is then left to putrefy, the sugar or nilk-sugar are found unaltered, even after the lapse of three months. It is evident, therefore, 
that these two substances, under the circumstances, cannot give rise to the formation of lactic acid, nor to that of acetic acid. The putrefaction of urea seems to be completely prevented by the presence of sugar, or milk-sugar; at least, in several experiments where one or the other of these two substances were present in the putrefying urine, the urea of the urine had not, even after the lapse of three months, acquired the property of effervescing with acids. I must here remark that a considerable amount of ammonia is formed in the process of putrefaction, owing to the resulting decomposition of another nitrogenous organic substance. This ammonia causes the protoxide of copper, formed upon testing the urine for milk-sugar with potass and sulphate of copper, to remain in solution, and to precipitate only upon the addition of acetic acid.

It follows from the preceding experiments and observations, that the organic acids in the urine of man are uric acid and hippuric acid, and another nitrogenous matter (most probably the colouring matter of urine), which latter substance, upon the access of air, resolves itself into acetic acid, and a substance resembling resin. Gay Lussac has already observed that it is only upon the access of air that urine passes into a state of putrefaction with absorption of oxygen*.

The considerations which I shall next adduce will prove sufficient to remove all doubt as to the cause of the acid reaction manifested by the urine of man.

It must be assumed as an irrefutable fact, that the inorganic bases present in urine, such as potass, soda, lime, \&c., have entered the organism through the medium of the aliments.

Now, in the first place, with respect to the nutriment of man, assuming it to consist of meat, that is, of animal substances and of aliments prepared from the flour of the cereals, or of the seeds of leguminous plants, such as peas, beans, and lentils, we know exactly the constitution of the ashes of flesh,

* Urine which, as is well known, speedily putreftes and passes from an ncid to an alkaline state, keeps very long in well-closed vessels, when it has scacely been allowed to come into contact with the air. It retains, in such case, its transparency, acidity, and odonr; it leposits only uric acid, and not the phosphate of ammonia and magnesia. If urine is left in contact with a suall amount of air it speedily absorbs the oxygen present, and decomposition then ceases; but if a sufficient amount of oxygen is present, a considerable amount of carbonate of ammonia is formed, and phosphate of lime and phosphate of magnesia and ammonia precipitate. It will be perceived that the decomposition of urine has no analogy in this respect with fermentation, since this latter process once set afloat continues without the co-operation of oxygen.-Gay Lussac, Axu. de Chimie, vol. Ixxvi. p. 245. 
as well as of the ashes of the cereals and the leguminous plants. There are no carbonated alkalies among the soluble salts of these ashes, but bibasic and tribasic phosphates of the alkalies, phosphate of soda or of potass, or both at the same time in varying properties. We might have supposed that the food in the stomach when in a state of solution would manifest the reaction of those salts which form the constituents of the ashes of the aliments partaken of. Now, in fact, all the bibasic and tribasic phosphated alkalies have an alkaline reaction, and yet nevertheless the reaction of the chyme is acid.

According to the best authenticated investigations, the acid reaction of the gastric juice is owing to the presence of free hydrochloric acid, and for the origin of this acid we must refer to common salt.

It is obvious that common salt undergoes decomposition in the organism, and becomes resolved into hydrochloric acid, which we find in a free state in the gastric juice, and into soda. This latter substance is, previous to the chyme having assumed the form adapted for its transformation into blood, that is, previous to its being changed into chyle, reconducted into the alimentary canal through the medium of the bile, the only compound of soda which we know with certainty to exist, as such, in the organism.

When we compare the composition of flesh, or of coagulated albumen, with that of the blood, we find a very considerable difference with regard to the inorganic constituents of these substances. The serum of the blood is miscible with water in all proportions, and has an alkaline reaction; the muscular fibre is insoluble, and has no alkaline properties. The ashes of blood contain principally alkaline phosphates, whilst the ashes of muscular fibre contain a far larger proportion of phosphate of lime. It is obvious that upon the transformation of the blood into muscular fibre, the greater amount of alkaline phosphates re-enter the circulation, whilst a certain amount of phosphate of lime remains in the organs in a state of chemical combination. When we consider that the insolubility of flesh, and of the cellular tissue in their natural state, depends upon the presence of the insoluble phosphate of lime, and that by the removal of the phosphate of lime the power of dissolving in alkaline fluids is restored to or increased in the remaining constituents containing nitrogen and sulphur, the necessity of the presence of free hydrochloric acid in the formation of chyme becomes immediately manifest. No mineral acid, and still less no organic acid, such as lactic acid or acetic acid, possesses the power of dissolving boiled meat 
and albumen, or any aliment similar to these, in the same degree as hydrochloric acid in the dilute state in which it exists in the gastric juice; and this dissolving power is, moreover, quite independent of the other substances present in the gastric juice, for those other substances do not act as solvents, they merely exercise an accelerating influence upon the process of solution. Hydrochloric acid in contact with calf's stomach, acts upon coagulated albumen simply with increased rapidity; the solution ensues with equal completeness, whether we use hydrochloric acid with calf's stomach, or pure hydrochloric acid diluted to the same extent. 'The difference between the processes in the two cases is simply a difference of time; the solution in the former case requires for its completion only the fifth part of the time necessary in the latter case; this fact has been confirmed by all the experiments on artificial digestion with access of air, and therefore of oxygen. The action of hydrochloric acid on bones will best illustrate the action which this acid exercises upon phosphate of lime. Microscopical investigations have shown us that the phosphate of lime is contained in bones in chemical combination with gelatine, and not deposited in gelatinous cells. When bones are digested in very dilute hydrochloric acid their phosphate of lime is dissolved out within a few per cents., and the residuary gelatine, which under other circumstances requires several hours for its solution in boiling water, now dissolves in the space of a few seconds.

Disregarding altogether the solvent power which hydrochloric acid exercises upon the animal substance itself, there is no doubt that it destroys the combination of the organic substance with phosphate of lime, and by that means increases its solubility.

When the hydrochloric acid (in the stomach) has exercised its solvent action upon the aliments, and the latter have passed into a state of solution, the soda, which originally entered the organism in combination with the hydrochloric acid, that is, as common salt, rejoins the hydrochloric acid during the preparation of the chyme, and previous to its transformation into chyle; the soda and the hydrochloric acid thus re-united combine again and form common salt. Chyle and lymph have no longer any acid reaction, but, on the contrary, they manifest alkaline properties.

The alkaline reaction of the lymph, chyle, and blood of man, and of the carnivorous animals, cannot be owing to the presence of a free alkali, as is evident from the preceding observations; for the nutriments of man, and of the carnivorous as well as the graminivorous animals, contain no free alkali, Phil. Mag. S. 3. Vol.25. No. 168. Dec. 184t. $2 \mathrm{H}$ 
nor any salt formed of an alkaline base and an acid which might be destroyed in the organism, by the vital process, and thus cause the alkaline base to be liberated. 'The blood must contain the same salts as exist in the aliments. With the exception of common salt, nothing is added during the digestion of the aliments. We have seen that this substance undergoes decomposition in the upper part of the digestive apparatus, being resolved into fiee soda and free hydrochloric acid; but we have also seen that the liberated soda rejoins the hydrochloric acid during the preparation of the chyme, and previous to the trangformation of the latter into chyle; that is, when the acia has performed its function, namely, the solution of the alimefts; the salt formed by this combination, that is, common salt, has neither an acid nor an alkaline reaction. The salts with alkaline reaction contained in meat, flour, or grain, are alkaline phosphates. It is obvious that the alkaline reaction of the chyle, lymph, and blood of animals feeding upon animal and vegetable substances, can only be derived from their alkaline phosphates. 'The serum of the blood ean only be considered as a combination of albumine with an alkaline phosphate; the fibrine of the blood, or the fibrine of the muscular fibre, is a combination of albumine with phosphate of lime.

The bibasic phosphates of soda and of potash are, in many respects, bighly remarkable salts; although of a tolerably strong alkaline reaction, yet they exercise no destructive action upon the skin or upon organic formations; they possess all the properties of the free alkalies without being such; thus, for instance, they absorb a large amount of carbonic acid, and this in such a manner that acids produce effervescence in a saturated solution of this kind, just as they would in alkaline carbonates; they dissolve coagulated curd of milk or cheese, as well as coagulated albumen, into clear fluids with the greatest facility, just as caustic or carbonated alkalies do. But of still greater importance in relation to the secretion of urine is their deportment towards hippuric acid and uric aciol. Hippuric acid dissolves with the greatest facility in water to which common phosphate of soda has been added; uric acid possesses the same property at a high temperature; the phosphate of soda, in this process, loses its alkaline reaction completely upon the addition of uric acid and hippuric acid, and assumes an acid reaction. The acid nature of the urine of man, and of the carnivorous and graminivorous animals, is thus explained in a very simple manner.

There are but two principal channels through which the salts entering the organism with the aliments can effect their 
exit from the body; viz. they must either be carried off in the freces or in the urine. The most simple experiments show that soluble salts are carried off by the fæces only when the amount of salt contained in the fluids in the intestines is larger than that contained in the blood; if the amount of salt in these fluids is equal or inferior to that of the blood, the soluble salts are re-absorbed by the absorbing vessels of the intestinal canal, and enter the circulation, and are then removed from the body by the urinary organs and channels. If the amount of salt contained in the intestinal canal is larger than that contained in the blood, the salts exercise a purgative action.

If, after previous evacuation of the rectum, a weak solution of common salt (one part of salt to sixty parts of water) be taken by means of a clyster, no second evacuation will take place; the fluid is absorbed, and all the salt is found in the urine. This experiment yields the most convincing results if ferrocyanide of potassium is substituted for common salt; in this case, the first urine excreted after the injection of the saline solution, and frequently even after so short a time as fifteen minutes, contains so copious an amount of ferrocyanide of potassium, as to yield, upon the addition of persalts of iron; a copious precipitate of prussian blue.

The influence which salts in general exercise upon the secretion of urine is in the highest degree worthy of attention. It is a well-known fact that $a$ very speedy emission of urine takes place, in healthy individuals, after drinking fresh pumpwater. If ten glasses of water, of from six to eight ounces each, containing no more than 1.500 th of its amount in salts, be drunk at short intervals, an emission of urine of the usual colour will, after the lapse of about ten minutes, follow the second glass, and from eight to nine evacuations of urine will generally occur in the course of an hour and a half. The urine, in this experiment, emitted in the last evacuation, will be clear and colourless, like pump-water, and the amount of salts it contains is little more than is contained in pumpwater. There are individuals who are capable of thus imbibing from six to eight quarts of water consecutively without any inconvenience.

But the case is quite different with water possessing an amount of salts equal to that of the blood; if even as little as 1-100th part of common salt be added to pump-water, and from three to four glasses drunk, no evacuation of urine will take place, even two hours after drinking. It is almost impossible to drink more than three glasses of this saline water, for it weighs heavily on the stomach, as if the absorbent vessels had no power of taking it up. This obviously arises from 
the fluid within the channels of circulation, i.e. the blood, and the fluid without these vessels, i.e. the saline water, not exercising any physical action upon one another, $i . e$. not intermixing by endosmose or exosmose.

Water containing a larger amount of salts than the blood, such as common sea-water, for instance, and even the weaker kinds of saline mineral waters, exercise again a different action from that of pump-water mixed with $1 \cdot 100$ th of common salt; not only no emission of urine takes place after the imbibition of such saline water, but water exudes from the circulating vessels into the intestinal canal, and, together with the saline solution, is carried off through the rectum; purgation takes place, attended with much thirst, if the saline solution be in some measure concentrated.

Considering that a certain amount of salts is absolutely necessary to constitute normal blood, we may deduce from these observations and experiments (which any one may easily imitate and verify upon his own person) that the physical condition of the tissues or of the blood-vessels opposes an obstacle to any increase or decrease of the amount of salts in the blood; and thus that the blood cannot become richer or poorer in salts beyond a certain limit.

Fluids containing a larger amount of salts than the blood remain unabsorbed, and leave the organism through the rectum; fluids containing a smaller amount of salts than the blood enter into the circulation, absorb, and remove from the organism, through the urinary channels, all the soluble salts and other substances which do not belong to the constitution of the blood; so that, finally, only those substances remain in the organism which exist in chemical combination with the constituents of the blood, and which, therefore, are incapable of being secreted by the healthy kidneys.

I have convinced myself, by careful and minute examinations, that urine emitted after drinking a copious amount of water invariably contains a somewhat larger amount of salts than the water which has been drunk; whilst the amount of phosphates contained in the last emitted portions of the urine is extremely minute, and no longer detectable by the ordinary tests. It is therefore obvious that all the salts, without exception, contained in the urine, are to be considered as accidental constituents of the blood, which are excreted and removed from the organism precisely because they no longer form part of the normal constitution of the blood. The phosphates emitted with the urine were, previously, constituents of substances which have been decomposed in the vital processes, or they existed as constituents of the blood, but upon its trans- 
formation into living tissues they were not admitted into their composition, they were not required in the constitution of the latter.

Now, among the products of the vital processes, which, together with the soluble phosphates, are removed from the organism through the urinary organs and channels, there are two organic acids, namely, uric acid and hippuric acid, both possessing the property of combining with the soda or potash of the alkaline phosphates, and acquiring in the combination a higher degree of solubility than they possess, per se, at the common temperature of the body. It is obvious that by the accession of these two acids, and by their action upon the phosphates of soda, an urate and a hippurate of soda must be formed on the one hand, and an acid phosphate of soda on the other; and that, consequently, the urine must acquire an acid reaction.

But the presence of these two acids in the urine is not the only cause of its acid nature; there exists another cause which tends powerfully to maintain and increase it.

According to the preceding remarks we ought to find in the urine all the soluble salts of the food, as well as a small amount of the phosphate of lime, which is soluble to a certain extent in acid fluids, together with magnesia. The amount of these latter substances will be in proportion to their solubility in acid phosphate of soda. The other insoluble salts of the aliments we ought to find in the fæces. In other words, assuming that the materials composing the aliments become converted into oxygen compounds, that is, are burnt in the organism, we ought to find in the urine all the soluble salts of their ashes, and in the fæces all the insoluble salts. Now, upon comparing the constitution of the ashes of the blood or of the aliments, or, rather, the salts contained therein, with those contained in the urine, we find that there exists a striking difference between their respective amount of sulphates.

According to the analyses of the ashes of the grains of wheat and rye*, the urine of an individual feeding exclusively upon bread ought not to contain a single trace of a sulphate, whilst the urine of an animal fed upon peas or beans ought to contain sulphates together with phosphates in the proportion of 9 of the former to 60 of the latter. Finally, as flesh contains no soluble alkaline sulphate (broth does not yield any precipitate of sulphate of barytes when tested with salts of barytes), the urine of carnivorous animals ought to be equally free from soluble sulphates. We find, on the con-

* Annalen der Chemie, vol. xlvi., p. 79. 
trary, that the urine of man, according to the most correct analyses, contains a far larger proportion of sulphates than the aliments partaken of ; nay, even that? the amount of the sulphuric acid received into the system must, in many cases, be equal or superior to that of the phosphoric acid contained in the aliments. According to the analysis of human urine made by Berzelius and Lehmann, the amount of the sulphates present in urine is nearly double that of all the soluble phosphates together. Hieronymi found the amount of sulphate of potash contained in the urine of the tiger, the lion, and the leopard, compared with that of the phosphates, to be as 1 to $7 \frac{1}{2}$. It can be distinctly and positively proved that these salts have not been partaken of in such proportions. But we now know the origin of the greatest portion of the sulphuric acid contained in the urine; this acid has entered the organism with the food, not in the form of a sulphate, but as sulphur.

Gluten*, vegetable casein, flesh, albumen, fibrine, and the cartilages and bones, contain sulphur in a form quite different from the oxygen compounds of this substance. This sulphur is separated as sulphuretted hydrogen during the putrefaction of these substances; it combines with the alkalies, operating upon these animal substances, and may be obtained from such combinations in the form of sulphuretted hydrogen by means of stronger acids.

Now we know, from the experiments of Wöhler, that the soluble sulphurets become oxidized in the organism; and that thus, for instance, sulphuret of potassium becomes converted into sulphate of potash; and it is therefore unquestionable that the sulphur of the constituents of the blood, derived from the aliments, or, what comes to the same point, the sulphur of the transformed tissues, becomes finally converted into sulphuric acid by the oxygen absorbed in the process of respiration, and thus that in the urine it must appear in the form of sulphates; and from this cause the original amount of these salts contained in the aliments becomes increased. The alkaline base which we find in the urine, in combination with this sulphuric acid, is supplied by the soluble alkaline phosphates; and the latter, in consequence of the loss of part of this base, are converted into acid salts.

By these considerations and views respecting the cause of the acid reaction of urine, I have been induced to prepare

* Dietrich has examined gluten (in the laboratory of Giessen) with regard to its amount of sulphur; he found wheat-gluten to contain from 0.033 per cent. to 0.035 per cent. of sulphur, exactly the same proportion as is contained in albumen or fibrine. 
an artificial urine, which possesses the properties of natural urine, even although sulphuric acid be altogether excluded.

If 40 grains of dry phosphate of soda (or 90 grains of the crystallized salt, $\mathrm{PO}_{5} 2\left\{\begin{array}{l}\mathrm{NaO} \\ \mathrm{HO}\end{array}+24 \mathrm{Aq}\right.$.) be dissolved in one pound of water, a fluid will be obtained having an alkaline reaction; if to this fluid we add 15 grains of uric acid, and 15 grains of hippuric acid, and the mixture is heated, both ncids will completely dissolve, imparting a strong acid reaction to the fluid. The solution thus prepared does not deposit a trace of uric acid at a temperature from $37^{\circ}-38^{\circ}$ $\left(=98^{\circ}, 100^{\circ}\right.$ Fahrenheit $=$ the heat of the blood $)$; nay, it is even only several hours after complete refrigeration that a sediment is formed, consisting of uric acid containing soda; this sediment is of an analogous form to that deposited by natural urine after standing at rest for a long time. Upon collecting this sediment, in one of my experiments, after the lapse of twenty-four hours, I found that it weighed $7 \frac{1}{2}$ grains, so that there remained still in solution $22 \frac{1}{2}$ grains of the organic acids. Dilute mineral acids produce immediately, in the fluids filtered off from the sediment, a precipitate of uric acid.

Proust, Prout, and all the other chemists who examined the urine previous to, or about the same period as Berzelius, ascribed its acid reaction to the uric acid or phosphoric acid; hippuric acid was not known as a constant attendant upon uric acid.

Proust says, at page 260 of the paper above referred to, " it is the phosphoric acid which imparts to the urine its acid properties. If urine is evaporated to dryness, then treated with alcohol, urea, the colvuring resinous principle, and phosphoric acid dissolve out; the two former substances exercise no action upon lime-water, but the phosphoric acid causes a white precipitate in lime-water, as well as in solutions of salts of lead; this precipitate is formed upon adding to lime-water, or to solutions of salt of lead, a few drops of the alcoholic solution."

With regard to lactic acid, Berzelius remarks, at page 4.21 of his Manual, "Lactic acid is a general product of the spontaneous destruction of organic matter within the organism, it is therefore contained in all the animal fluids. It is formed in the largest proportion in the muscles, becomes saturated by the alkali of the blood, and, in the kidneys, is again separated from the alkali. It is principally this acid which forms the free acid of the urine; and although this contains acid phosphate of ammonia and acid phosphate of lime, yet these 
substances owe their origin and formation to the circumstance of the lactic acid sharing the bases with the phosphoric acid.

"In the course of an investigation into the constitution of urine, in the year 1807, I found lactic acid as a constituent of urine; this acid had not, up to that period, been counted among the constituents of urine; and several chemists having, on very slight grounds, asserted it to be acetic acid, I submitted it to the test of a new examination; the results of this examination led me to conclude that lactic acid could not be confounded with acetic acid, and that the acid found was lactic acid, and not acetic acid.

"The lactic acid contained in the urine is the principal solvent for the phosphate of lime; this may be easily seen from the fact, that after having extracted from exsiccated urine the free lactic acid by means of alcohol, the residue indeed contains acid phosphates, but upon redissolving it in water it leaves the greatest portion of the phosphate of lime as an insoluble residue."

I deem it scarcely necessary to examine the reasons which induced Berzelius, thirty-seven years ago, to assume the presence of lactic acid (a substance the properties of which were then totally unknown) in urine and in animal fluids. The then prevailing idea that lactic acid nust be a product of the decomposition of all animal fluids, because it is formed in milk, has now turned out to be totally unfounded. Milk contains neither lactic acid nor lactates, the formation of lactic acid in milk being dependent upon milk-sugar.

It was evidently from entertaining these incorrect notions that Berzelius was led to assume the presence of lactic acid in certain non-volatile fluids of the urine, which have an acid reaction, and contain organic substances beside phosphoric acid; there is no doubt that the presence of lactic acid in these fluids was highly probable, if lactic acid were truly a constant product of the putrefaction of all animal fluids. But when we consider that the properties of lactic acid were then so little known that many chemists even disputed the fact of its existence, and asserted it to be nothing more than acetic acid in disguise, we see clearly that there could exist no analytical grounds, at that time, whereon to found a positive assertion of its presence in urine and other animal fuids. At present there is far less reason for such a supposition, since now we have closely examined and studied this acid, and have obtained a correct knowledge of its properties, and yet we are incapable of detecting even a trace of it in any animal fluid.

In the nineteenth volume of Poggendorff's Annalen der 
Physite und Chemie, p. 26, Berzelius says, "Leopold Gmelin, whose lucid researches occupy so distinguished a place in animal chemistry, has almost put himself at the liead of those who positively deem lactic acid to be acetic acid."

Proust and other chemists had asserted the organic acid contained in urine to be acetic acid, and had thereby induced Berzelius to examine more minutely the properties of lactic acid, in order to determine its difference from acetic acid. Thus, twenty-three years after the supposed detection of lactic acid in urine, the very existence of this acid remained still a matter of doubt.

Now, although Berzelius states (p. 422 of his work) that the assertion of the presence of acetic acid in urine, on the part of other chemists, induced him to enter into a comparative examination of lactic acid and of acetic acid, this examination was not made, as one would suppose it would have been, with the organic acid of the urine, asserted by him to be lactic acid, but, on the contrary, with real lactic acid produced from milk; this investigation proved the existence of lactic acid in milk, but it did not follow from this that lactic acid is present in urine, since, as I just now remarked, milk had been used for these experiments and not urine. [No lactic acid can be produced from broth by the method recommended by Berzelius.]

Finally, concerning Berzelius's view, that lactic acid is the solvent of the phosphate of lime in the urine, and that this substance becomes insoluble whenever the free lactic acid is removed by alcohol, we need merely recollect the well-known property of acid phosphate of lime to be resolved by alcohol into phosphoric acid, which dissolves in the alcohol, and into the common insoluble bone-earth, to perceive at once the fallacy of this inference. We know that it is precisely upon this property that the separation of phosphoric acid from lime depends. Alcohol dissolves phosphoric acid and hippuric acid out of urine evaporated to dryness, but no lactic acid.

After the preceding remarks, it will not be difficult to appreciate the true value of the evidence upon which the assumption of the presence of lactic acid in urine and in animal fluids depends; if I add that the method followed by Berzelius in his experiments (decomposition of urea by boiling with milk of lime, \&c.) can no longer be considered perfectly exact and correct, it will be admitted that, so far as our present experience and observations enable us to judge, no kind of putrefaction, such, for instance, as the formation of lactic acid, can take place in the organs of the living and healthy animal body.

When,-notwithstanding the esteem I have always enter- 
tained, and shall always entertain, for my highly-honoured friend whose views I am controverting, - I thus enter into a searching and critical investigation respecting lactic acid, both historically and analytically, it is for the interest of science, and to ascertain the truth upon this highly important subject. The notion of the presence of lactic acid in the animal body, in the gastric juice, in the milk, in the blood, in the urine, is so extensively diffused, and the part which has been assigned to this acid in digestion, in the respiratory process, \&c., is so great and comprehensive, that the proof of its non-presence and, consequently, of the impossibility of assigning to it any share whatever in the mysterious processes of the animal organism, was well deserving of being supported by every admissible reason.

The diffusion of lactic acid, or of that acid which was assumed to be the lactic, in the animal body, depends, moreover, only upon the delusion existing, from the beginning, with regard to this acid. Berzelius states (p. 460 of his work), when alluding to Hieronymi's analysis of the urine of the lion, in which that chemist asserted that he had found acetic acid, "I have taken the liberty here to substitute the term lactic acid for acetic acid, for reasons which will be apparent from the preceding remarks." Now, the reason for this substitution was, that Berzelius, in an experiment made for this especial purpose, had found that fresh urine yields no acetic acid upon distillation with a mineral acid,-a fact which, as already stated, I have had occasion to confirm by my own experiments. This substitution of laetic acid for acetic acid, where the presence of the latter had been asserted previously, was, in the subsequent editions of his Manual, consistently carried through in all the statements of analyses of animal matter. The circumstance that in milk, as well as in sour-krout, upon acidification, acetic acid is invariably formed, besides lactic acid, renders the propriety of this substitution the more doubtful, since the acetic acid was thus of course wholly disregarded.

It follows, from all we have hitherto stated, that the acid nature of the urine of carnivorous animals, as well as of that of man, depends upon the nature of the bases partaken of in the aliments, and upon the particular form of their conbinations. In the flesh, blood, and other parts of animals, as well as in the grains of the cereal and leguminous plants, there exists no free alkali. The alkali which these substances contain is invariably combined with phosphoric acid: the acids formed in the organism by the vital process, namely, sulphuric acid, hippuric acid, and uric acid, share the alkali amongst them, 
and this of course must give rise to the liberation of a certain amount of phosphoric acid, or what comes to the same point, to the formation of a certain amount of acid phosphates of soda, lime, and magnesia. The proportional amount of the liberated phosphoric acid varies with the temperature; at a higher temperature the phosphate of soda dissolves a larger amount of uric acid and hippuric acid than at a lower temperature, -at from $37^{\circ}-38^{\circ}$ more than at $15^{\circ}$. It is owing to this that urine, upon refrigeration, sometimes deposits uric acid, or urate of soda in a crystalline state, which of course can only take place by the uric acid, at a lower temperature, restoring to the phosphoric acid the soda or potash which at a higher temperature it had withdrawn from it. At the common temperature phosphoric acid decomposes urate of soda, whilst at a higher temperature uric acid decomposes phosphate of soda. When urine, containing uric acid and manifesting an acid reaction, forms no sediment upon cooling, it shows that the amount of the phosphoric acid and that of the uric acid exactly balance each other with regard to their affinity for soda. Had there been present a larger proportion of uric acid, this would have separated upon cooling; whilst, on the other hand, the presence of a preponderating proportion of phosphoric acid would likewise have caused the precipitation of uric acid, because the affinity of the former for soda would then exceed that of the latter. This explains the circumstance that urine in certain states, when, from some cause or other, its amount of sulphuric, hippuric, or other acid becomes increased, precipitates a larger proportion of uric acid than urine in its normal state. The solubility of uric acid in urine must decrease in proportion as the amount of the other acids present in the urine increases, because those acids share the soda with the uric acid; and of course the larger the amount of soda which combines with these other acids the less comes to the share of the uric acid. It is likewise owing to this that uric acid very frequently precipitates from urine upon the addition of mineral or other acids, and that urine of a turbid, whey-like appearance, from the presence of uric acid, frequently manifests a far more strongly acid reaction than normal urine.

Now, bearing in mind that the use of alkaline citrate (Gilbert Blane), of neutral paratartrate of potash, bi-tartrate of potash, tartarized soda, acetates of potash and soda, and tartarized borax, renders the urine alkaline by creating in it an amount of carbonated alkali; and that, likewise, after the eating of fruit, such as cherries, strawberries, \&c., the urine is of an alkaline nature, inasmuch as these fruits contain alka- 
lies combined with vegetable acids, it is obvious that the acid reaction of healthy urine is purely accidental, and that urine of an alkaline or neutral reaction cannot be considered as a symptom of a diseased condition of the body. All vegetable aliments, without exception, tubers, roots and leaves, potatoes, turnips, greens, \&c., contain alkalies in combination with vegetable acids; potatoes, for instance, contain alkaline citrates; turnips, alkaline racemates and oxalates, \&c. All these plants yield, upon incineration, more or less strongly alkaline ashes, the bases of which were contained in the living plants as salts of vegetable acids.

It is obvious that by adding these vegetables to a meat diet, to bread and to other aliments prepared from flour, the nature of the urine must become thoroughly altered; for the alkalies which these vegetables contain in combination with vegetable acids, enter the urine, in the form of carbonated alkalies, and neutralize the acids, of whatever kind, which may be present. When partaken of in a certain proportion they render the urine neutral ; when partaken of in a larger proportion they impart to it an alkaline reaction.

The urine of all animals feeding upon vegetables, such as grass, herbs, roots, \&c., has an alkaline reaction. The urine of the horse, of the cow, of the sheep, of the camel, of the rabbit, of the guinea-pig, of the ass, \&c., is alkaline; it contains alkaline carbonates, and acids produce in it a lively effervescence.

The acid, neutral, or alkaline reaction of urine of healthy individuals does not depend upon any difference in the processes of digestion, respiration, or secretion, in the various classes of animals, but upon the constitution of the aliments, and upon the alkaline bases which enter the organism through the medium of these aliments. If the amount of these bases is sufficiently large to neutralize the acids formed in the organism, or supplied by the aliments, the urine is neutral; whilst it manifests an alkaline reaction when the amount of alkaline bases thus supplied to the organism is more than sufficient to neutralize the acids; but in all these cases the urine accords with the nature of the aliments taken.

The inorganic bases and acids contained in the urine were, with the exception of sulphuric acid, which joins them in the organism, constituents of the aliments. The amount of inorganic bases and acids emitted through the urine in twentyfour hours must, in adult individuals, be equal to that of these bases and acids supplied to the organism, during the same period, through the medium of the aliments.

From these data, it follows necessarily,-first, that the ana- 
lysis of urine, when made without respect to the inorganic salts, acids and bases supplied by the aliments, teaches nothing whatever, and by no means justifies us in drawing therefrom any physiological or pathological inference; secondly, that from the nature of the ashes of the aliments we are able to determine, positively, the constituents of the urine emitted; and thirdly, that only when these latter have been distinctly ascertained can we expect to derive, from the analysis of the urine, any correct information with respect to the inorganic matters which have come to be present in it through processes of disease ; this, at least, is the chemical method of quantitative investigation.

Bearing in mind that the urine contains the soluble constituents of the ashes of the aliments, whilst the fæces contain the insoluble part of these constituents, we may from an accurate knowledge of both, at once determine in which urine soluble alkaline phosphates must be present, and in which they cannot exist. The ashes of all seeds, and of flesh and blood, contain a certain amount of soluble and insoluble phosphates, whilst the ashes of vegetables contain no free alkaline phosphate, but only insoluble phosphates. 'These vegetable ashes contain far more lime and magnesia than is required for the neutralization of the phosphoric acid present. Hence, upon incinerating a plant, together with its seed, and lixiviating the ashes, we find in the fluid obtained no alkaline phosphate, although the lixivium of the ashes of the seeds, when incinerated and lixiviated by themselves, yields a considerable amount of these phosphates; the excess of lime and magnesia contained in the leaves and the straw enter here into combination with the phosphoric acid of the soluble alkaline phosphates, forming an insoluble compound.

It will now be understood why the alkaline phosphates are generally absent from the urine of herbivorous animals, and also why, in certain cases, they may be found in the urine of these animals. If the nutriment of these animals contains no soluble phosphates, their urine cannot contain any, whilst if we add a certain proportion of grain to their food, the alkaline phosphates may be detected in their urine. Thus it is obvious, likewise, that the soluble phosphates in the urine of man are merely accidental constituents, and that by simply adding lime or magnesia to the aliments, and thus assimilating the constitution of these aliments to that of the food of herbivorous animals, the urine must become altered in its nature and properties. 'The knowledge of the influence which alkalies, magnesin and lime, or acids, exercise upon the properties of the urine, or, in other words, upon the secretory pro- 
cess of the kidneys, in the healthy organism, is of the highest importance for the curing of diseases.

$I$ believe that there is now required only a small number of good and correct observations to establish a fixed rule for the remedies necessary in various cases. Future properlydirected experiments will prove whether sanguification is absolutely dependent upon the presence of alkaline phosphates or not; we shall be able to determine whether weak solutions of alkaline phosphates are not the best solvents for uric acid deposited in the bladder; and likewise what is the infuence which aliments rich in sulphur, such as mustard, for instance, exercise upon the separation of uric acid in the bladder, in consequence of the formation of sulphuric acid. At any rate, we may, by a judiciously selected diet, alter with positive certainty, and at pleasure, the nature of the urine; we may, without cansing any injury to health, keep it alkaline for a long time by adopting a vegetable diet; and this is certainly the first condition necessary to ensure the entire prevention of the formation of uric acid, as is the case with the herbivorous animals. By its combination with an alkaline base, uric acid must in the organism resolve itself into its ultimate oxygen compounds with the same facility as other organic acids, if the physician prohibits all substances to be taken as food which, like wine or fat, take possession of the oxygen necessary for the transformation of uric acid into carbonic acid and urea.

The carbonated alkali in the urine of herbivorous animals is separated from the blood by the kidneys; the urine derives it from the blood; it is certain, therefore, if we examine the blood one hour or a few hours after the animal has partaken of food, we must find in it this alkali in the same state as it is found in the urine, and that at other periods of the day the ashes of the blood may not contain the least trace of free alkali. But the fiee alkali does not cooperate in the vital process in the animal organism; or, if it is necessary in this process, the part which it has to perform may be undertaken with the very same effect by the bibasic and tribasic alkaline phosphates.

In like manner, when we are contemplating the presence of hydrochloric acid in the gastric juice, we must remember that the alkaline bases, soda, potash, lime, magnesia, are present in the aliments whilst in their natural state, invariably in the form of salts; that is, in combination with phosphoric acid or with organic acids. When, therefore, in the digestive process, hydrochloric acid is supplied by the gastric juice, the first action of this acid is confined to the decomposition of these salts; 
the hydrochloric acid withdraws lime from the phosphate of lime; potash, or soda, chloride of calcium, or chloride of sodium or potassium, is formed on the one hand, and acid phosphate of soda or potash, or acid phosphate of lime, on the other; or acetic acid, paratartaric acid, or citric acid, are liberated by the decomposition of the salts of these vegetable acids contained in the aliments. At a certain stage of digestion the chyme will, according to the nature of the food partaken of, contain acid phosphates or free vegetable acids; and it is only upon the supply of gastric juice continuing, that thus, upon the amount of the hydrochloric acid increasing, we may detect, by analysis, free hydrochloric acid in the chyme; the gastric juice taken from an empty stomach contains invariably free hydrochloric acid or acid phosphates.

With respect to the essential organic constituents of urine, I have already treated of the origin of urea and uric acid in my "Chemistry applied to Physiology and Pathology ;" and I will here merely add a few words on the presence of ammonia in urine.

Ammonia is a product of the putrefaction of nitrogenous substances, and as such might be a purely accidental constituent of the healthy animal body, and of its secretions. In consequence of certain processes which take place in the organism independently of the vital process, all the fluids in the body may of course become rich in ammonia, or in ammoniacal salts. Healthy urine contains only very minute or doubtful traces of ready-formed ammonia, and these traces existed probably already in the food partaken of. Fresh urine evolves ammonia when treated with alkalies, but it yields no precipitate with chloride of platinum. Dr. Schlossberger made certain experiments to this effect in the laboratory at Giessen; upon treating fresh urine with chloride of platinum, and allowing the mixture to stand at rest during the night, crystals were formed in the urine, which, upon examination, manifested all the properties of chloride of platinum and potassium. The amount of ammonia formed in the bealthy organism is likewise very minute, not being sufficient even to neutralize the acid from which proceeds the acid reaction of urine and of saliva. We cannot assume the presence of any ammoniacal salt in the urine of herbivorous animals, which contains fixed or alkaline carbonates.

The carbonate of ammonia which Chevreul found as a constituent of the urine of the camel, was either a product of putrefaction, or an accidental constituent of this urine, since the carbonate of ammonia is a volatile substance, and must find 
the skin and lungs a more direct way for its exit from the organism than the kidneys.

Experiments for the determination of the amount of ammonia in the urine of healthy individuals may become of importance in judging of pathological states; for in fevers, and other diseases, the amount of ammonia in the urine increases considerably. It is possible that, by analysing the urine, we may, in the increasing or decreasing amount of ammonia, obtain a measure for the alterations which take place in diseases. But the salts of potash, which are rarely absent, as well as the ammonia which is formed by the action of chloride of platinum upon the organic constituents of urine, render this reagent (the chloride of platinum) very unsafe for determining the increasing or decreasing amount of ammonia in the urine during disease. The magnesia salts would perhaps answer this purpose better; the quantitative examinations made with salts of magnesia are inferior to those made with chloride of platinum, but they are exact enough for the purpose of comparison.

\section{Intelligence and Miscellaneous Articles.}

ON THE ESSENTIAL OIL OF BETULA LENTA AND ON GUALTHERINE. BY W. PROCTER.

THE author has found that the essential oil, which is obtained 1 by the distillation with water of the Betula lenta (sweet birch or mountain mahogany), which is so common in North America, and which gives to some of its flowers their agreeable odour, which resembles that of gualtheria oil, is identical with the latter; but that the bark evolves this odour only when in contact with water, whilst originally it contains an inodorous body only, viz. gualtherine, which is converted into the essential oil when water is present, by the reaction of another substance also present (analogous to synaptase or emulsine), exactly as the oils of bitter almonds and mustard are formed.

By the action of barytic water, this gualtherine yields a new acid, the gualtheric acid. Unfortunately the author has not made use of elementary analysis.

Oil of Betula lenta.-This oil smells and tastes like gualtheria oil, has a specific gravity of $1 \cdot 173$, becomes reddened by the action of the atmosphere, but loses the colour on distillation; is but little soluble in water, in all proportions in alcohol and æther; produces a purple-red colour with protosalts of iron ; forms crystalline compounds immediately with solution of potash, soda, baryta and oxide of lead; these yield the oil unaltered when treated with dilute sulphuric acid; when treated with excess of potash, salicylic acid is formed; it is slowly dissolved by ammonia, and the solution deposits crystals of salicylamide. Crystalline products are obtained by the action of chlorine and bromine, hydrochloric and bromic acids being 